DO-TH 2004/13

December 2004

\title{
Probing the Perturbative NLO Parton Evolution in the Small- $x$ Region
}

\author{
M. Glück, C. Pisano, E. Reya \\ Universität Dortmund, Institut für Physik, \\ D-44221 Dortmund, Germany
}

\begin{abstract}
A dedicated test of the perturbative QCD NLO parton evolution in the very small- $x$ region is performed. We find a good agreement with recent precision HERA-data for $F_{2}^{p}\left(x, Q^{2}\right)$, as well as with the present determination of the curvature of $F_{2}^{p}$. Characteristically, perturbative QCD evolutions result in a positive curvature which increases as $x$ decreases. Future precision measurements in the very small $x$-region, $x<10^{-4}$, could provide a sensitive test of the range of validity of perturbative QCD.
\end{abstract}


Parton distributions $f\left(x, Q^{2}\right), f=q, \bar{q}, g$, underlie a $Q^{2}$-evolution dictated by perturbative $\mathrm{QCD}$ at $Q^{2} \gtrsim 1 \mathrm{GeV}^{2}$. It was recently stated [1] that the NLO perturbative QCD $Q^{2}$-evolution disagrees with HERA data 2, 3] on $F_{2}^{p}\left(x, Q^{2}\right)$ in the small- $x$ region, $x \lesssim 10^{-3}$. In view of the importance of this statement we perform here an independent study of this issue. In contrast to [1] we shall undertake this analysis in the standard framework where one sets up input distributions at some low $Q_{0}^{2}$, here taken to be $Q_{0}^{2}=1.5$ $\mathrm{GeV}^{2}$, corresponding to the lowest $Q^{2}$ considered in [1, and adapting these distributions to the data considered. In the present case the data considered will be restricted to

$$
1.5 \mathrm{GeV}^{2} \leq Q^{2} \leq 12 \mathrm{GeV}^{2}, \quad 3 \times 10^{-5} \lesssim x \lesssim 3 \times 10^{-3}
$$

as in [1] and will be taken from the corresponding measured $F_{2}^{p}\left(x, Q^{2}\right)$ of the H1 collaboration [2]. The choice of these data is motivated by their higher precision as compared to corresponding data of the ZEUS collaboration [3], in particular in the very small $-x$ region.

We shall choose two sets of input distributions based on the GRV98 parton distributions [4. In the first set we shall adopt $u_{v}, d_{v}, s=\bar{s}$ and $\Delta \equiv \bar{d}-\bar{u}$ from GRV98 and modify $\bar{u}+\bar{d}$ and the gluon distribution in the small $-x$ region to obtain an optimal fit to the $\mathrm{H} 1$ data [2] in the aforementioned kinematical region. We shall refer to this fit as the 'best fit'. The second choice will be constrained to modify the GRV98 $\bar{u}+\bar{d}$ and $g$ distributions in the small- $x$ region as little as possible. We shall refer to this fit as $\mathrm{GRV}_{\text {mod }}$. It will turn out that both input distributions are compatible with the data to practically the same extent, i.e. yielding comparable $\chi^{2} / d o f$. In view of these observations we do not agree with the conclusions of ref. [1], i.e. we do not confirm a disagreement between the NLO $Q^{2}$-evolution of $f\left(x, Q^{2}\right)$ and the measured [2, 3] $Q^{2}$-dependence of $F_{2}^{p}\left(x, Q^{2}\right)$.

The remaining flavor-singlet input distributions at $Q_{0}^{2}=1.5 \mathrm{GeV}^{2}$ to be adapted to 
the recent small- $x$ data are expressed as

$$
\begin{aligned}
x g\left(x, Q_{0}^{2}\right) & =N_{g} x^{-a_{g}}\left(1+A_{g} \sqrt{x}+7.283 x\right)(1-x)^{4.759} \\
x(\bar{u}+\bar{d})\left(x, Q_{0}^{2}\right) & =N_{s} a^{-a_{s}}\left(1+A_{s} \sqrt{x}-4.046 x\right)(1-x)^{4.225}
\end{aligned}
$$

where the parameters relevant for the large $x$-region, $x>10^{-3}$, which is of no relevance for the present small- $x$ studies, are kept unchanged and are taken from, e.g. GRV98 [4]. The refitted relevant small- $x$ parameters turn out to be

$$
\begin{aligned}
& \text { 'best fit' }: N_{g}=1.70, \quad a_{g}=0.027, \quad A_{g}=-1.034 \\
& N_{s}=0.171, \quad a_{s}=0.177, \quad A_{s}=2.613 \\
& \mathrm{GRV}_{\text {mod }}: N_{g}=1.443, \quad a_{g}=0.125, \quad A_{g}=-2.656 \\
& N_{s}=0.270, \quad a_{s}=0.117, \quad A_{s}=1.70
\end{aligned}
$$

to be compared with the original GRV98 parameters [4]: $N_{g}=1.443, a_{g}=0.147$, $A_{g}=-2.656$ and $N_{s}=0.273, a_{s}=0.121, A_{s}=1.80$. The resulting predictions are compared to the H1-data [2] in Fig. 1. These results are also consistent with the ZEUSdata [3] with partly lower statistics. The corresponding $\chi^{2} / d o f$ are 0.50 for the 'best fit' $(d o f=48)$ and 0.94 for $\operatorname{GRV}_{\text {mod }}(d o f=50)$, respectively. Our treatment of the heavy flavor contributions to $F_{2}$ differs from that in [1]. We evaluate these contributions in the fixed flavor $f=3$ scheme of [4], together with the massive heavy quark $(c, b)$ contributions, rather than in the $f=4$ (massless) scheme utilized in [1. We have checked, however, that our disagreement with [1] does not result from our $f=3$ plus heavy quarks vs. the $f=4$ massless quark calculations in [1]: we have also performed a fit for $f=4$ massless quarks and the results for $F_{2}$ and its curvature, to be discussed below, remain essentially unchanged.

In Figs. 2 and 3 we show our gluon and sea input distributions in (2) and (3), as well as their evolved shapes at $Q^{2}=4.5 \mathrm{GeV}^{2}$ in the small- $x$ region. It can be seen that both 
of our new small- $x$ gluon distributions at $Q^{2}=4.5 \mathrm{GeV}^{2}$ conform to the rising shape obtained in most available analyses published so far, in contrast to the valence-like shape obtained in [1] where the gluon density $x g$ decreases as $x \rightarrow 0$. It is possible to conceive a valence-like gluon at some very-low $Q^{2}$ scale, as in [4, but even in this extreme case the gluon ends up as non valence-like at $Q^{2}>1 \mathrm{GeV}^{2}$, in particular at $Q^{2}=4.5 \mathrm{GeV}^{2}$, as physically expected.

Turning now to the curvature test of $F_{2}$ advocated and discussed in [1, we first present in Fig. 4 our results for $F_{2}\left(x, Q^{2}\right)$ at $x=10^{-4}$, together with two representative expectations of global fits [5, 6], as a function of [1]

$$
q=\log _{10}\left(1+\frac{Q^{2}}{0.5 \mathrm{GeV}^{2}}\right)
$$

This variable has the advantage that most measurements lie along a straight line [1] as indicated by the dotted line at $x=10^{-4}$ in Fig. 4. The MRST01 parametrization [5] results in a sizable curvature for $F_{2}$ in contrast to all other fits shown in Fig. 4. This large curvature, incompatible with the data presented in [1, is mainly caused by the valencelike input gluon distribution of MRST01 at $Q_{0}^{2}=1 \mathrm{GeV}^{2}$ in the small- $x$ region which becomes even negative for $x<10^{-3}$ [5]. A similar result was obtained in [1] based on a particular gluon distribution $x g\left(x, Q^{2}\right)$ which decreases with decreasing $x$ for $x \lesssim 10^{-3}$ even at $Q^{2}=4.5 \mathrm{GeV}^{2}$ (cf. fig. 7 in [1]). More explicitly the curvature can be directly extracted from

$$
F_{2}\left(x, Q^{2}\right)=a_{0}(x)+a_{1}(x) q+a_{2}(x) q^{2} .
$$

The curvature $a_{2}(x)=\frac{1}{2} \partial_{q}^{2} F_{2}\left(x, Q^{2}\right)$ is evaluated by fitting the predictions for $F_{2}\left(x, Q^{2}\right)$ at fixed values of $x$ to a (kinematically) given interval of $q$. In Fig. 5(a) we present $a_{2}(x)$ which results from experimentally selected $q$-intervals [1]:

$$
\begin{aligned}
& 0.7 \leq q \leq 1.4 \text { for } 2 \times 10^{-4}<x<10^{-2} \\
& 0.7 \leq q \leq 1.2 \text { for } 5 \times 10^{-5}<x \leq 2 \times 10^{-4} \\
& 0.6 \leq q \leq 0.8 \text { for } \quad x=5 \times 10^{-5}
\end{aligned}
$$


Notice that the average value of $q$ decreases with decreasing $x$ due to the kinematically more restricted $Q^{2}$ range accessible experimentally. For comparison we also show in Fig. 5(b) the curvature $a_{2}(x)$ for an $x$-independent fixed $q$-interval

$$
0.6 \leq q \leq 1.4 \quad\left(1.5 \mathrm{GeV}^{2} \leq Q^{2} \leq 12 \mathrm{GeV}^{2}\right) .
$$

Apart from the rather large values of $a_{2}(x)$ specific for the MRST01 fit as discussed above (cf. fig. 4), our 'best fit' and GRV $_{\text {mod }}$ results, based on the inputs in (4) and (5), respectively, do agree well with the experimental curvatures as calculated and presented in [1] using $\mathrm{H} 1$ data. It should be noted that perturbative NLO evolutions result in a positive curvature $a_{2}(x)$ which increases as $x$ decreases. This feature is supported by the data shown in fig. 5(a); since the data point at $x<10^{-4}$ is statistically insignificant, future precision measurements in this very small $x$-region should provide a sensitive test of the range of validity of perturbative QCD evolutions.

Furthermore, the H1 collaboration [2] has found a good agreement between the perturbative NLO evolution and the slope of $F_{2}\left(x, Q^{2}\right)$, i.e. the first derivative $\partial_{Q^{2}} F_{2}$.

To conclude, the perturbative NLO evolution of parton distributions in the small- $x$ region is compatible with recent high-statistics measurements of the $Q^{2}$-dependence of $F_{2}^{p}\left(x, Q^{2}\right)$ in that region. A characteristic feature of perturbative QCD evolutions is a positive curvature $a_{2}(x)$ which increases as $x$ decreases (cf. fig. 5). Although present data are indicative for such a behavior, they are statistically insignificant for $x<10^{-4}$. Future precision measurements and the ensuing improvements of the determination of the curvature in the very small $x$-region should provide further information concerning the detailed shapes of the gluon and sea distributions, and perhaps may even provide a sensitive test of the range of validity of perturbative QCD.

This work has been supported in part by the 'Bundesministerium für Bildung und Forschung', Berlin/Bonn. 


\section{References}

[1] D. Haidt, Eur. Phys. J. C35, 519 (2004)

[2] C. Adloff et al., H1 Collab., Eur. Phys. J. C21, 33 (2001)

[3] S. Chekanov et al., ZEUS Collab., Eur. Phys. J. C21, 443 (2001)

[4] M. Glück, E. Reya, A. Vogt, Eur. Phys. J. C5, 461 (1998)

[5] A.D. Martin, R.G. Roberts, W.J. Stirling, R.S. Thorne, Eur. Phys. J. C23, 73 (2002)

[6] J. Pumplin et al., JHEP 7, 12 (2002) hep-ph/0201195 


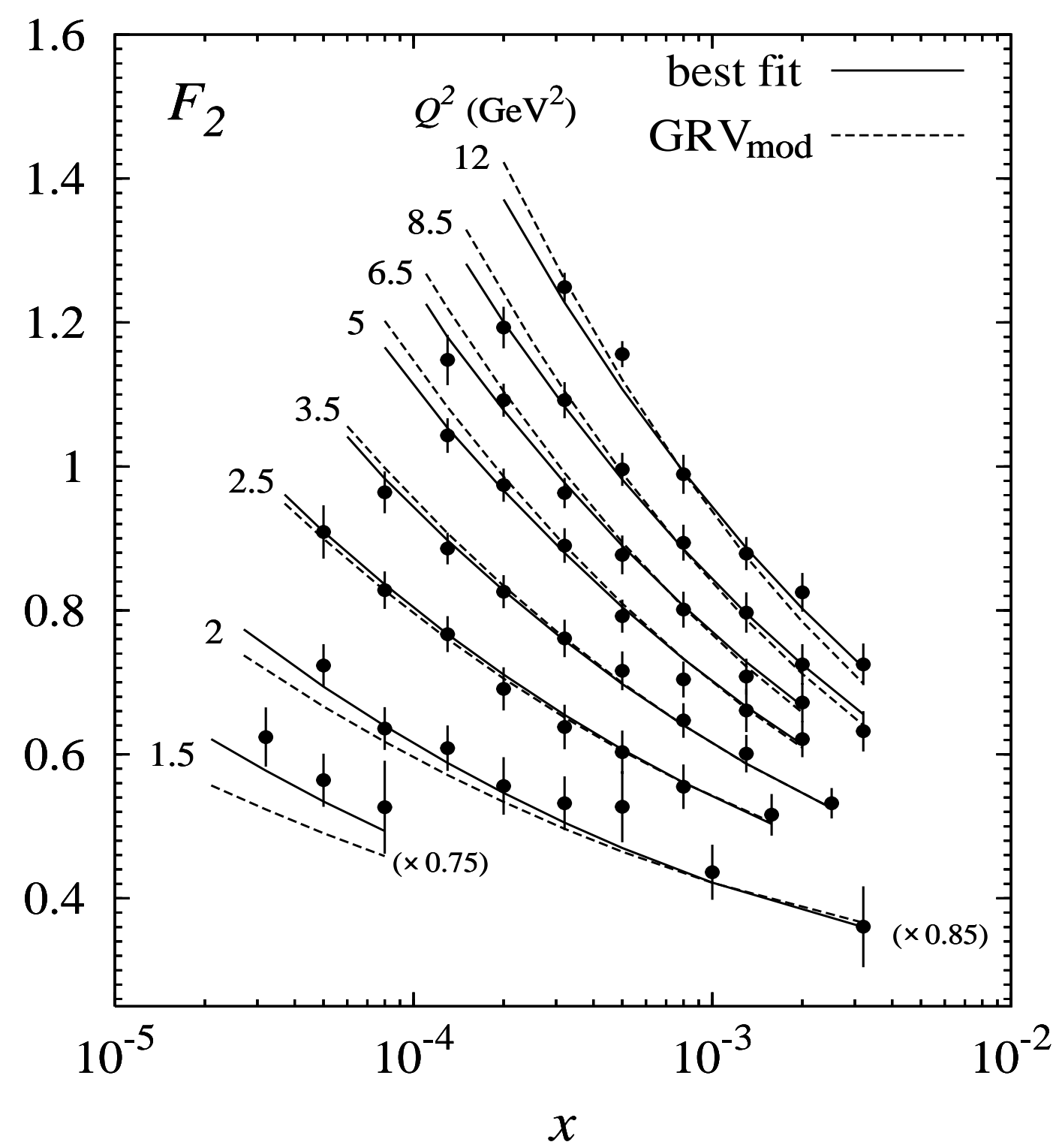

Figure 1: Comparison of our 'best fit' and GRV $_{\text {mod }}$ results based on (4) and (5), respectively, with the $\mathrm{H} 1$ data [2]. To ease the graphical representation, the results and data for the lowest bins in $Q^{2}=1.5 \mathrm{GeV}^{2}$ and $2 \mathrm{GeV}^{2}$ have been multiplied by 0.75 and 0.85 , respectively, as indicated 


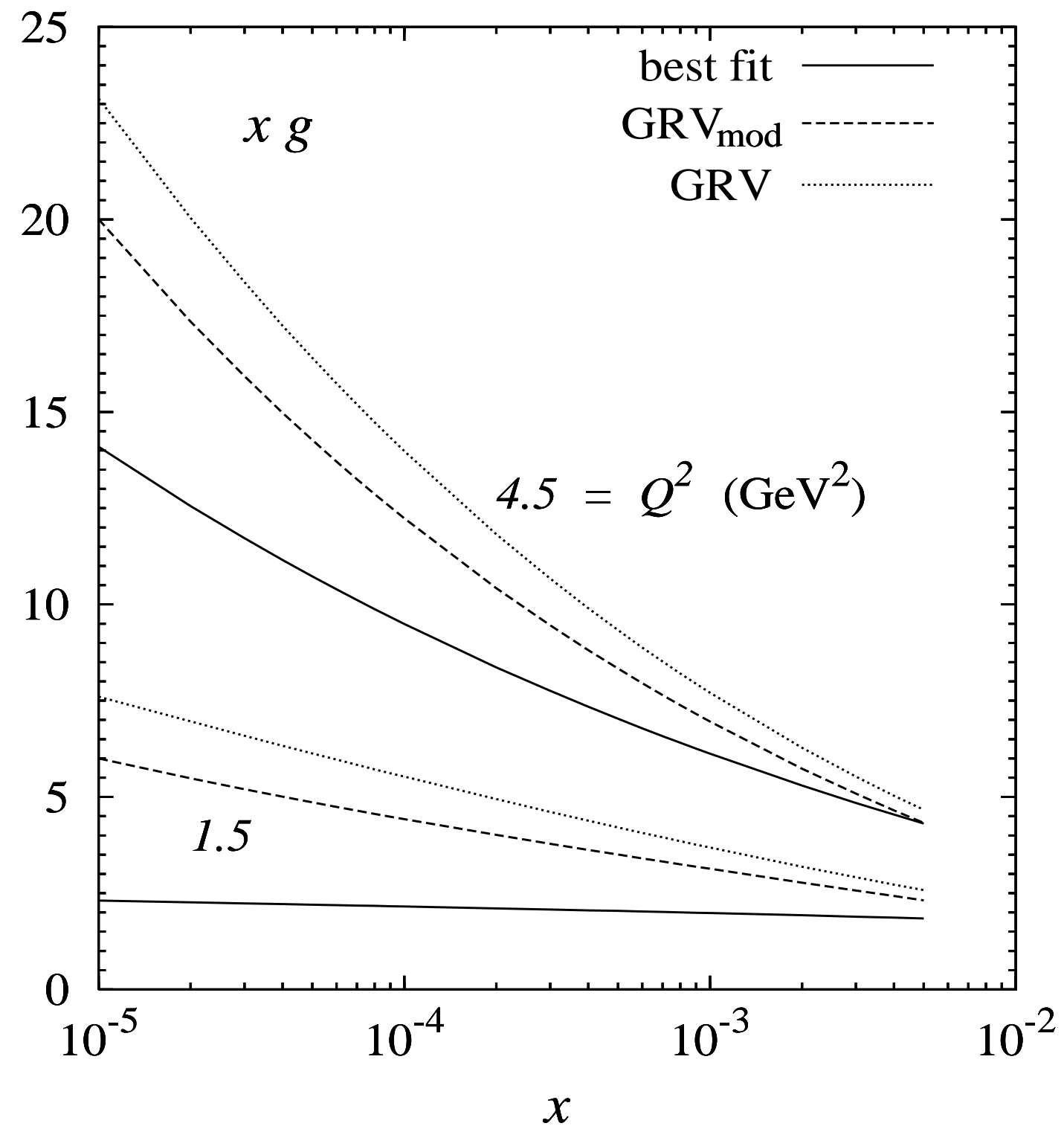

Figure 2: The gluon distributions at the input scale $Q_{0}^{2}=1.5 \mathrm{GeV}^{2}$ corresponding to (2)

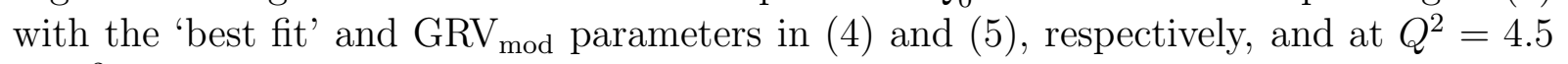
$\mathrm{GeV}^{2}$. For comparison, the original GRV98 results 4] are shown as well by the dotted curves 


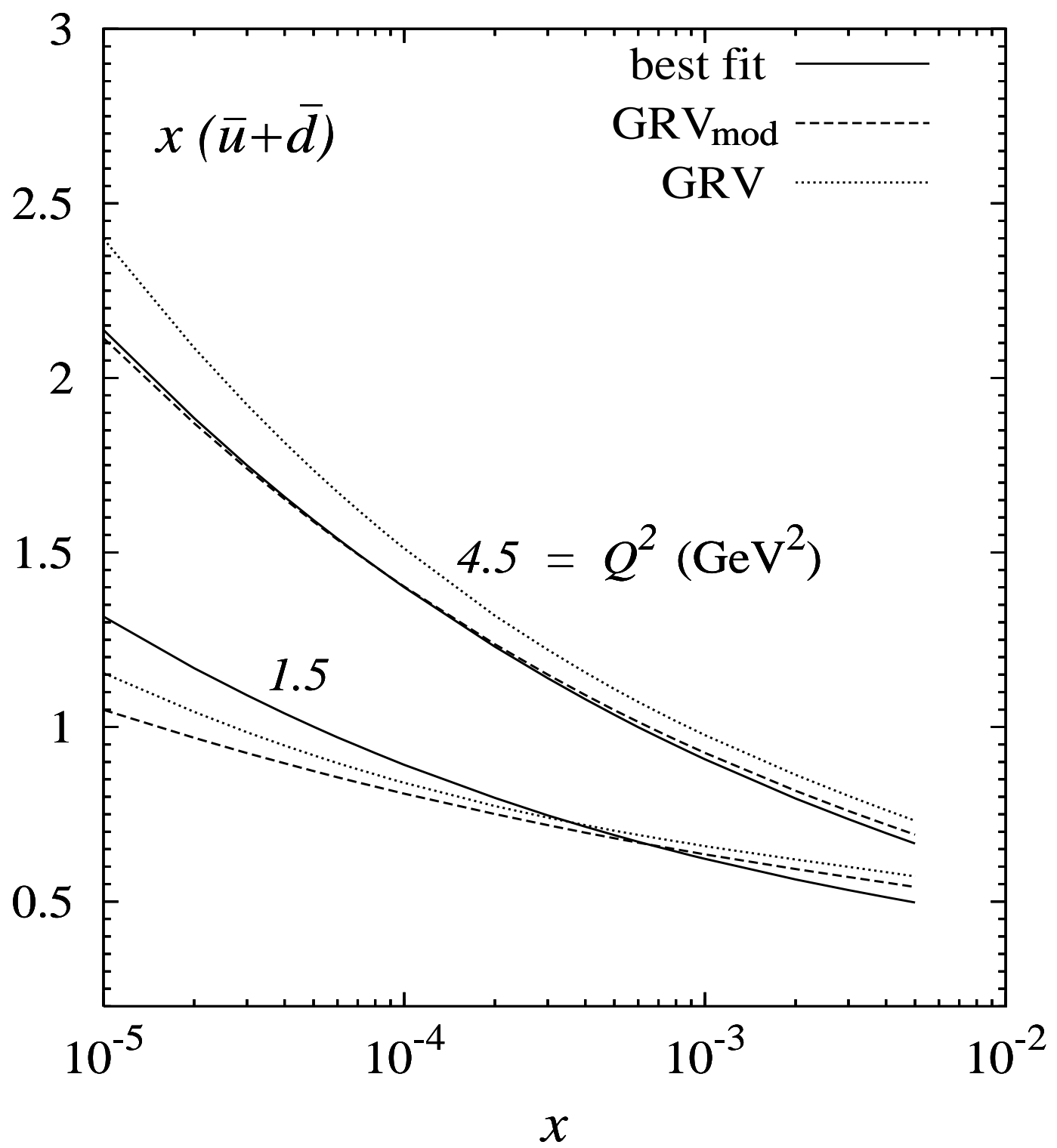

Figure 3: The sea distribution $x(\bar{u}+\bar{d})$ at the input scale $Q_{0}^{2}=1.5 \mathrm{GeV}^{2}$ in (3) with the 'best fit' and $\mathrm{GRV}_{\text {mod }}$ parameters in (4) and (5), respectively, and at $Q^{2}=4.5 \mathrm{GeV}^{2}$. For comparison, the original GRV98 results [4] are shown as well by the dotted curves 


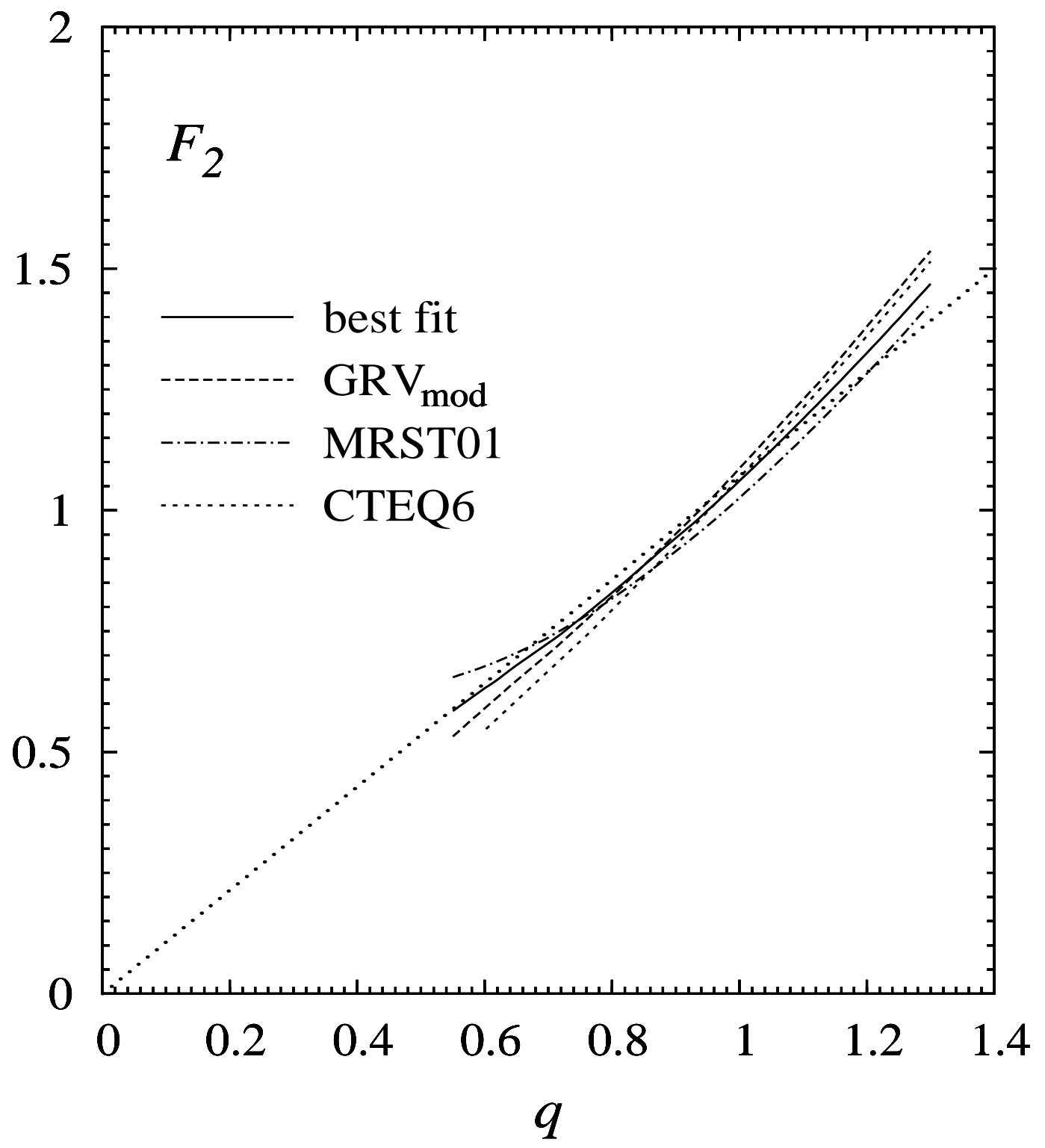

Figure 4: Predictions for $F_{2}\left(x, Q^{2}\right)$ at $x=10^{-4}$ plotted versus $q$ defined in (6). Representative global fit results are taken from MRST01 [5] and CTEQ6M [6]. Most small- $x$ measurements lie along the straight (dotted) line [1] 

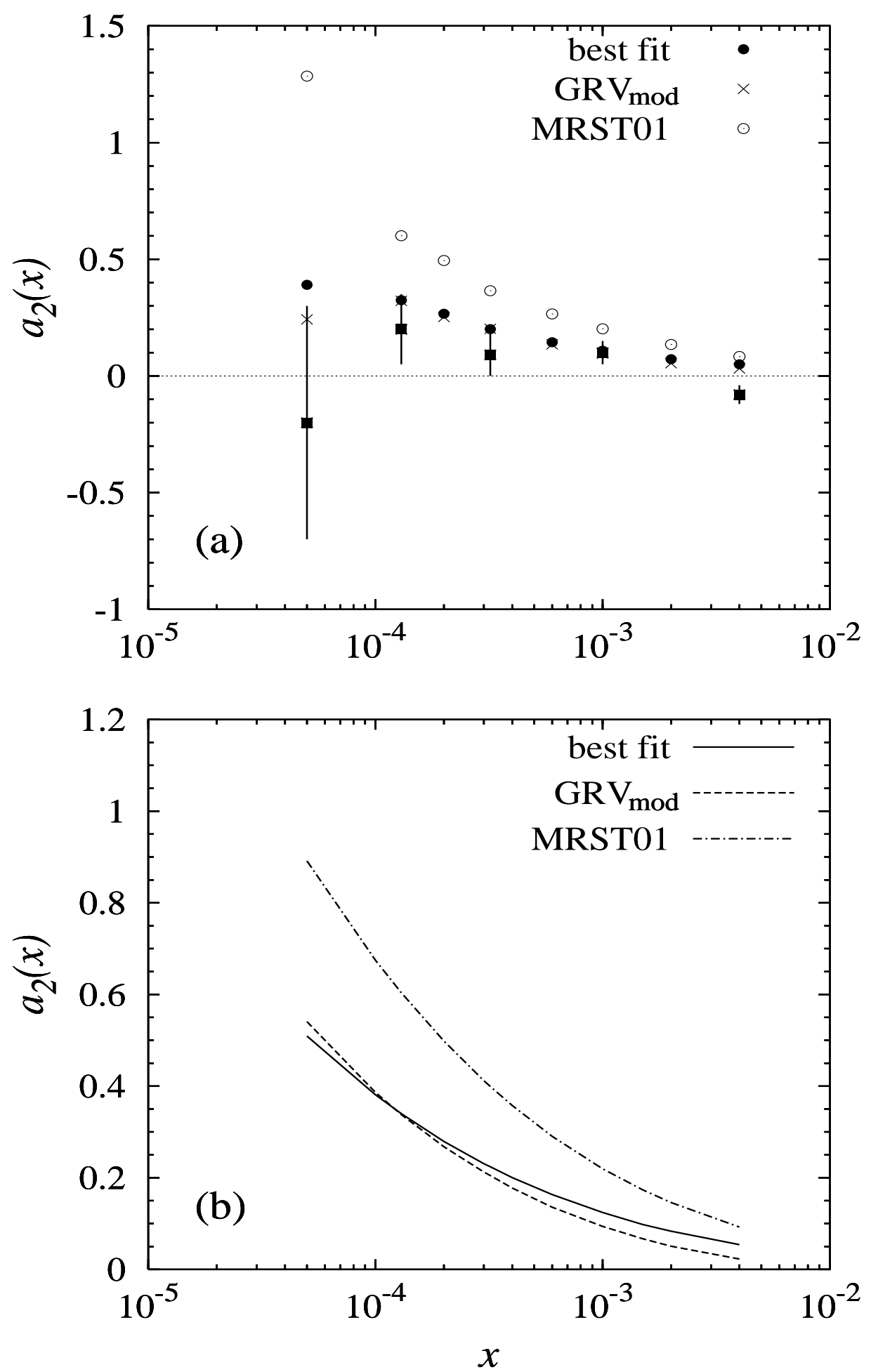

Figure 5: The curvature $a_{2}(x)$ as defined in (7) for (a) the variable $q$-intervals in (8) and (b) the fixed $q$-interval in (9). Also shown are the corresponding MRST01 results [5]. The experimental curvatures (squares) shown in (a) are taken from [1] 